\title{
Characterizing Optical Constants of Thin Films for Vacuum Ultraviolet Lithography Applications
}

\author{
Wonder FAn, Hsuen-Li Chen ${ }^{1, *}$, Chi-Lung WU ${ }^{2}$ and Kuei-Shu Chang-LiaO ${ }^{2}$ \\ National Nano Device Lab., 1001-1 Ta Hsueh Road Hsinchu, Taiwan, R.O.C. \\ ${ }^{1}$ Department of Materials Science and Engineering, National Taiwan University, Taipei, Taiwan, R.O.C. \\ ${ }^{2}$ Institute of Engineering and System Science, National Tsing Hua University, Hsinchu, Taiwan, R.O.C. \\ (Received October 31, 2003; accepted January 6, 2004; published June 29, 2004)
}

The determination of optical constants of thin films is an important requirement for the study of masks, antireflective coatings, and resist materials used in the vacuum ultraviolet (VUV) lithography process. We demonstrate a simple method for determining optical constants by measuring the reflectance of thin films with different incidence angles. In order to obtain accurate optical constants, the measurement of reflectance, incidence angles and thickness should be confirmed carefully. In addition, thickness measurements are not important for thick high-absorption films. The measured reflectance should be modified by the surface roughness of thin films particularly in vacuum ultraviolet regimes. The optical constants of various materials were obtained experimentally by this method without using complicated mathematics models.

[DOI: $10.1143 / \mathrm{JJAP} .43 .3684]$

KEYWORDS: vacuum ultraviolet, optical constants, thin films, reflectance, roughness

\section{Introduction}

In the recent journals of International Technology Roadmap for Semiconductors (ITRSs), optical lithography has been gradually shifted from the deep ultraviolet (DUV) $(248 \mathrm{~nm}$ and $193 \mathrm{~nm})$ to the vacuum ultraviolet (VUV) regimes $(157 \mathrm{~nm}$ and $121 \mathrm{~nm})$, which will lead integratedcircuit (IC) technologies to the generation of sub-70 nm. ${ }^{1,2}$ ) Determining the optical constants (refractive index and extinction coefficient) is important for the analysis of newly developed materials at the exposure wavelength. However, most thin films (such as those used in resists, phase shift materials, and as top and bottom anti-reflective coating materials) and substrates (such as silicon and fused silica) are not transparent in vacuum ultraviolet regimes and their optical constants cannot be determined by the conventional reflectance-transmission (R-T) method. ${ }^{3)}$ An alternative method is using a commercialized VUV ellipsometer for the characterization of optical constants. However, a VUV ellipsometer has a complicated configuration, is expensive and its working wavelength is generally larger than $150 \mathrm{~nm}$. Therefore, it is difficult to analyze optical constants below $150 \mathrm{~nm}$, such as the 126 or $121 \mathrm{~nm}$ nodes using a VUV ellipsometer. ${ }^{4,5)}$

Here we demonstrate a simple method, which can be applied in vacuum ultraviolet lithography for determining optical constants of thin films by measuring reflectance with different incidence angles. ${ }^{6}$ Because the measurement tolerances are critical in this method, the reflectance, incidence angle and thickness should be determined carefully. The thickness and roughness of thin films were measured using a common visible ellipsometer and an atomic force microscope (AFM), respectively. As a result, the measured reflectance can be modified by the surface roughness of thin films to obtain accurate optical constants. We found that the optical constants of various types of materials could be determined by this method in vacuum ultraviolet regimes.

*E-mail address: hlchen@ndl.gov.tw

\section{Experimental Setup}

Different types of materials were prepared on silicon substrates, including low-absorption (e.g. silicon oxide), semi-absorption (e.g. silicon nitride) and high-absorption (e.g. titanium nitride) materials by high-density-plasma chemical vapor deposition (HDP-CVD) system (BR2000LL), and using a sputter system (ULVAC SBH-3308 $\mathrm{RDE})$. The thickness and surface roughness of thin films were measured using an ellipsometer (SOPRA SE-5) and AFM (Digital Instruments Nano Scope II), respectively. ${ }^{7)}$ Reflectance spectra were measured using a deep and vacuum ultraviolet optical spectrometer (Acton Research Co.), which has a spectral range from 120 to $300 \mathrm{~nm}$ and incidence angles ranging from 15 to $60 \mathrm{deg}$.

\section{Analytic Method}

Here we illustrate the analytic method, which is based on the thin film theory. ${ }^{8)}$ For example, we demonstrate how to determine the optical constants of a silicon nitride film with a thickness of $83 \mathrm{~nm}$. First, the curved surfaces of reflectance dependent on various sets of optical constants for a silicon nitride film were simulated at different incidence angles as shown in Fig. 1(a). Then the measured reflectances with different incidence angles were plotted on the curved surfaces. In order to obtain the optical constants, the measured isoreflectance curves on the surfaces of reflectance can be projected on the optical-constants plane as shown in Fig. 1(b). Therefore, the optical constants of silicon nitride films can be obtained from the intersection of two projective isoreflectance curves. In addition, to obtain a unique and accurate solution of optical constants, it is essential to reduce the tolerances of measurements.

\section{Result and Discussions}

Due to measurement errors, a variety of tolerances for characterizing optical constants should be considered in this method. The tolerances for reflectance, thickness and incidence angle are discussed for low-absorption, semiabsorption and high-absorption materials. In order to acquire a unique set of optical constants, the measured reflectance 


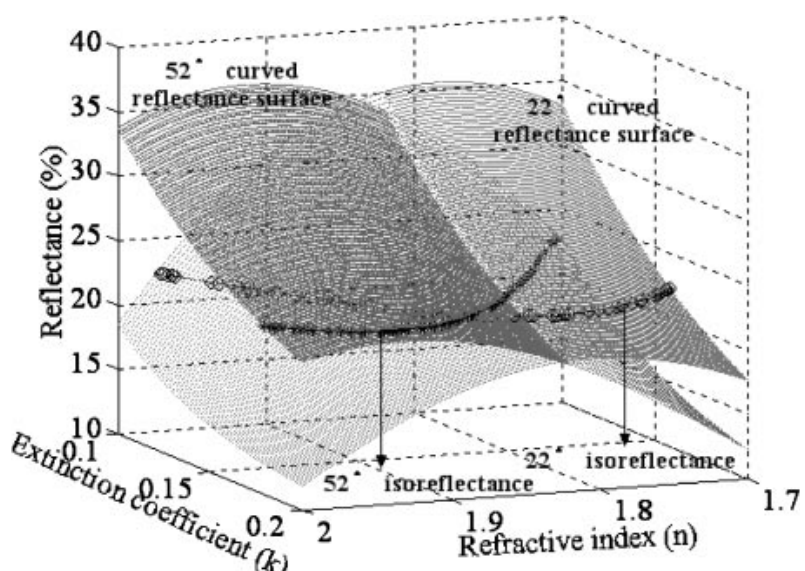

(a)

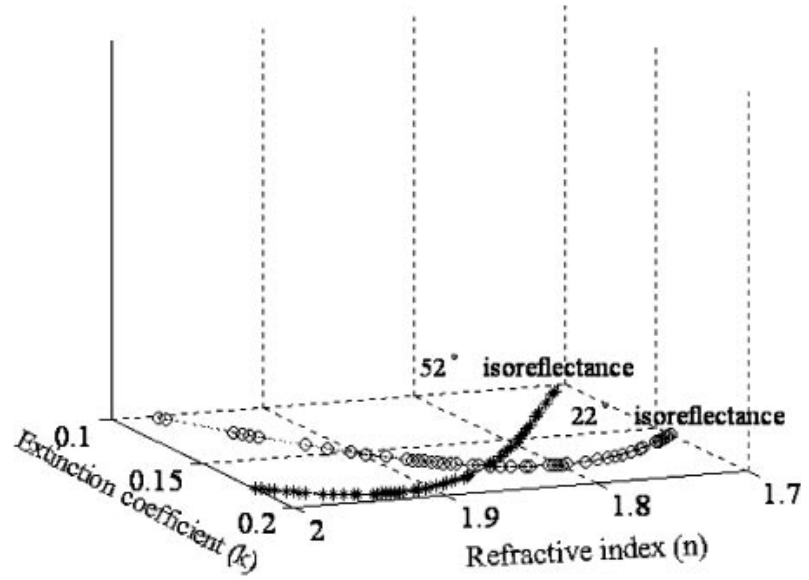

(b)

Fig. 1. (a) Curved surfaces of reflectance and isoreflectance curves dependent on optical constants with different incidence angles (b) The optical constants can be obtained from the intersection of projected isoreflectance curves.

should be determined for at least three different angles. The tolerances for reflectance, thickness and incidence angle are controlled within $1 \%, 1 \mathrm{~nm}$ and $1^{\circ}$, respectively. To quantify the range of possible optical constants, the deviation factor is defined as $\left(\Delta n^{2}+\Delta k^{2}\right)^{1 / 2}$ for the analysis of optical constants varies with the measured tolerances. The $\Delta n$ and $\Delta k$ are the deviations of refractive index and extinction coefficient respectively, caused by measurement tolerance.

\subsection{Reflectance tolerances analysis}

The ranges of possible optical constants calculated from reflectance tolerances are shown in Figs. 2(a)-2(c) for lowabsorption (silicon oxide), semi-absorption (silicon nitride) and high-absorption (titanium nitride) materials. The calculated deviation factors are $0.051,0.018$ and 0.086 for lowabsorption, semi-absorption, high-absorption materials respectively. Results show that the reflectance measurement should be confirmed carefully particularly for low-absorption and high-absorption materials.

\subsection{Thickness tolerance analysis}

Similarly, the ranges of possible optical constants calculated from thickness tolerances are shown in Figs. 3(a)-3(c) for silicon oxide, silicon nitride and titanium nitride films.

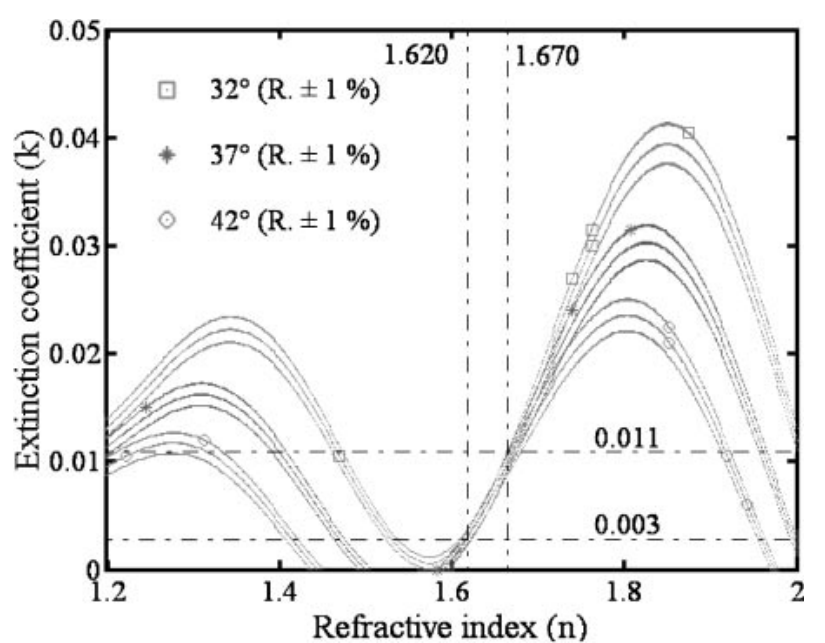

(a)

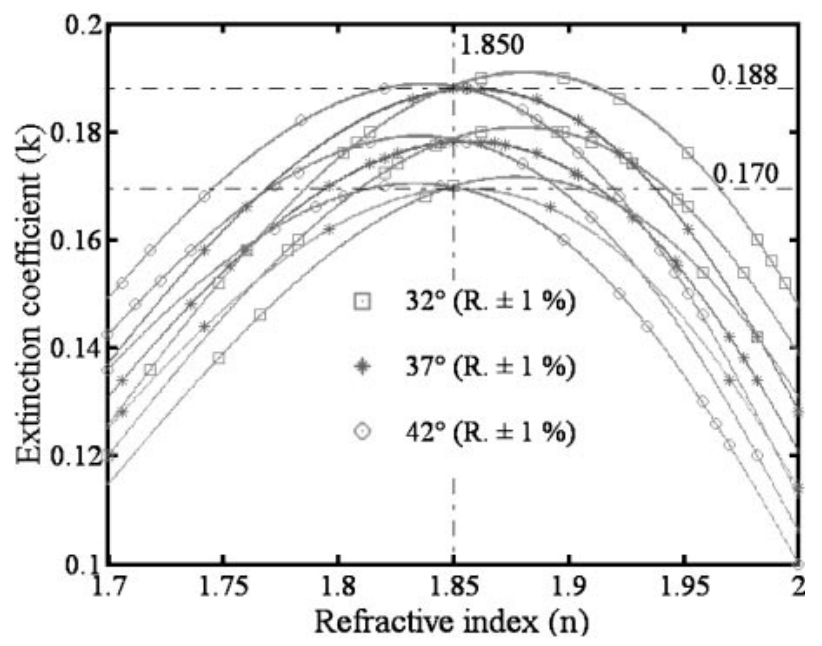

(b)

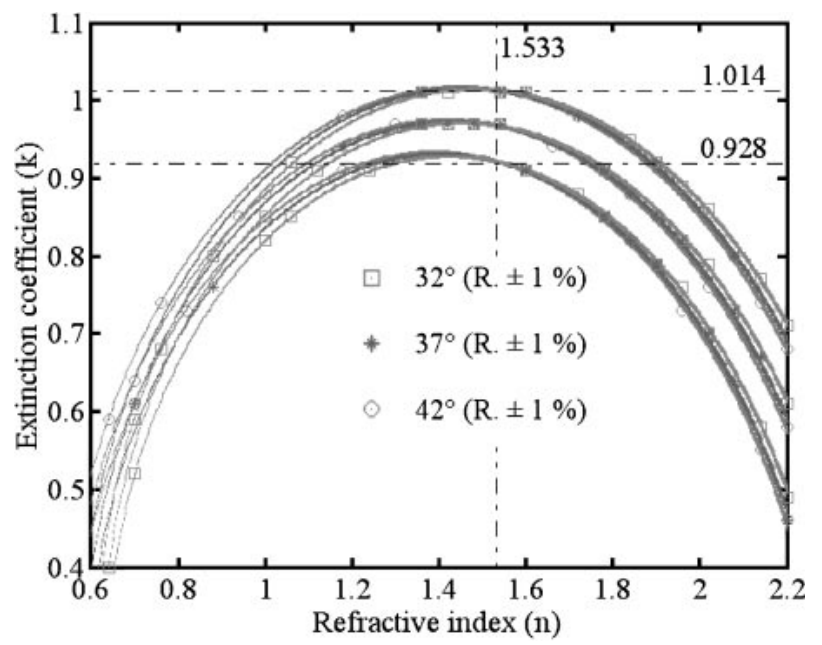

(c)

Fig. 2. Ranges of possible optical constants calculated from reflectance tolerances for (a) low-absorption (b) semi-absorption and (c) highabsorption materials.

The calculated deviation factors are $0.069,0.048$ and 0.001 for low-absorption, semi-absorption, and high-absorption materials, respectively. Results show that the thickness measurement should be affirmed carefully particularly for 


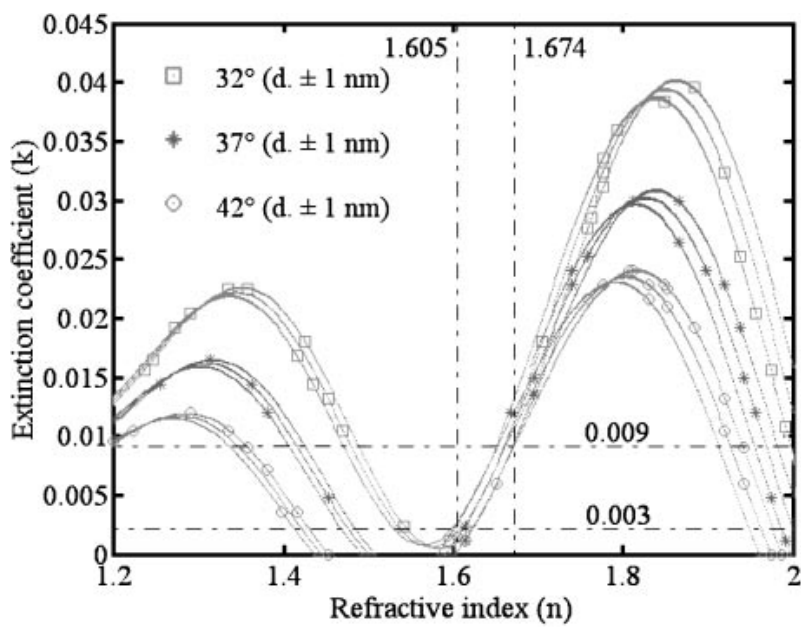

(a)

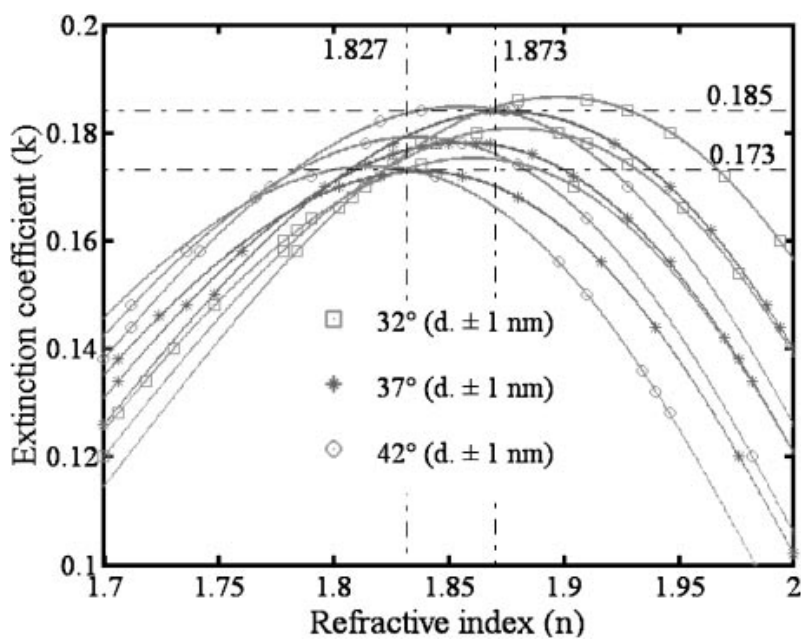

(b)

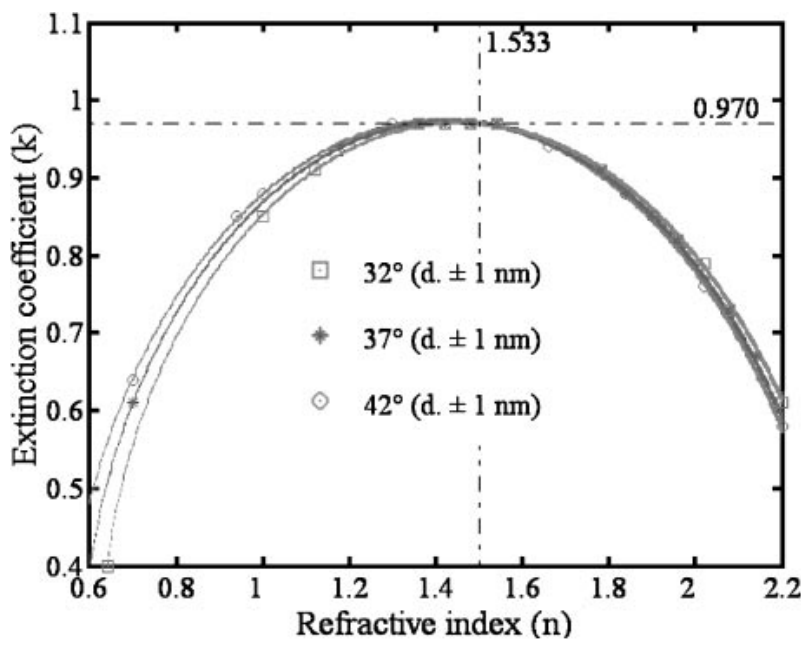

(c)

Fig. 3. Ranges of possible optical constants calculated from thickness tolerances for (a) low-absorption (b) semi-absorption and (c) highabsorption materials.

low-absorption and semi-absorption materials to obtain an accurate solution. In addition, thickness tolerance is not important for thick high-absorption films, because the reflectance varies slowly with the thickness of highabsorption films.

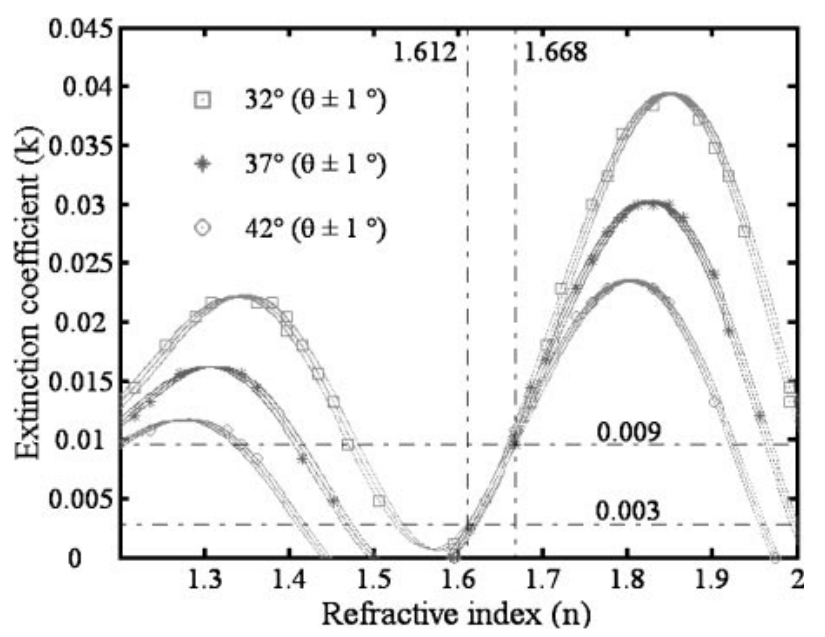

(a)

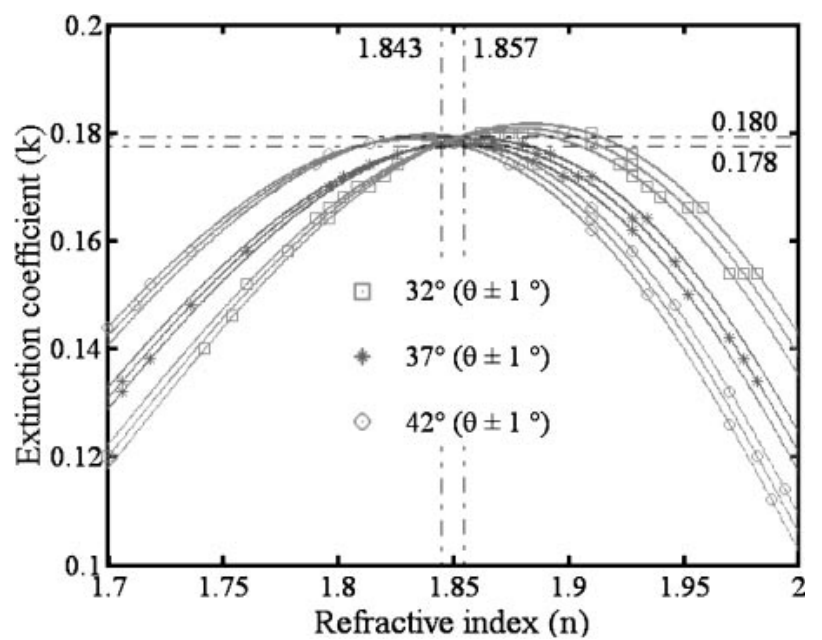

(b)

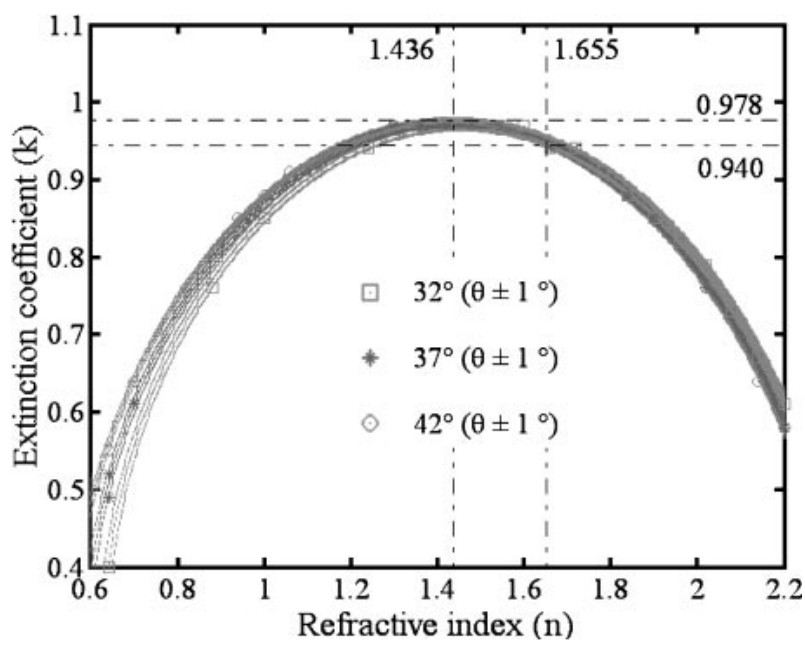

(c)

Fig. 4. Ranges of possible optical constants calculated from incidence angle tolerance for (a) low-absorption (b) semi-absorption and (c) highabsorption materials.

\subsection{Incidence angle tolerance analysis}

The ranges of possible optical constants resulting from thickness tolerances are shown in Figs. 4(a)-4(c) for silicon oxide, silicon nitride and titanium nitride films. The 


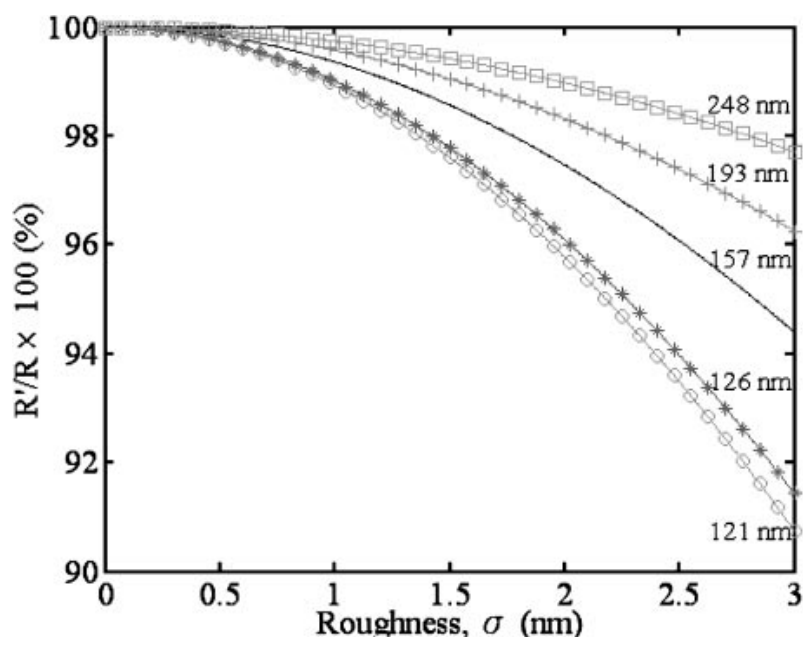

Fig. 5. Reflectance reduced by surface roughness of thin films at different lithography wavelengths.

calculated deviation factors are $0.056,0.014$ and 0.222 for low-absorption, semi-absorption and high-absorption materials, respectively. Results show the incidence angles should be affirmed carefully particularly for low-absorption and high-absorption materials to obtain an accurate solution. Therefore, decreasing all the measurement tolerances is an effective way to obtain accurate optical constants for various materials.

\subsection{Reflectance reduction caused by surface roughness of films}

In general, reflectance is reduced by the surface roughness of thin films particularly in short-wavelength regimes, which would result in inaccurate measurement of reflectance. It is therefore important to measure the surface roughness of a thin film to modify the measured reflectance. The reduced ratio of reflectance caused by roughness is defined by eq. (1). ${ }^{7)}$

$$
R^{\prime} / R \times 100 \%=\exp \left[-(4 \pi \sigma / \lambda)^{2}\right] \times 100 \%
$$

where $R^{\prime}$ is the reduced reflectance caused by roughness, $R$ is the ideal reflectance without roughness, $\sigma$ is the surface roughness of thin film, and $\lambda$ is the incidence wavelength. Figure 5 shows the reflectance reduction ratio $\left(R^{\prime} / R\right)$ for the range of surface roughness from 0 to $3 \mathrm{~nm}$ with different wavelengths. Results indicate that the reflectance is reduced more markedly in VUV regimes than in the longer lithography wavelength regimes. As a result, the measured reflectance should be modified by the surface roughness of thin films to obtain the accurate reflectance.

\subsection{Experimental results of different materials}

In order to obtain the optical constants of different types of materials, the key factor is to reduce and modify measurement tolerances described previously. First, for the low-absorption materials, the measured surface roughness and thickness of a silicon oxide film were $2.363 \mathrm{~nm}$ and $128 \mathrm{~nm}$, respectively. As a result, the reasonable optical constants obtained were about $(1.650,0.008)$ at $157 \mathrm{~nm}$ from the intersection of different reflectance curves shown in Fig. 6.

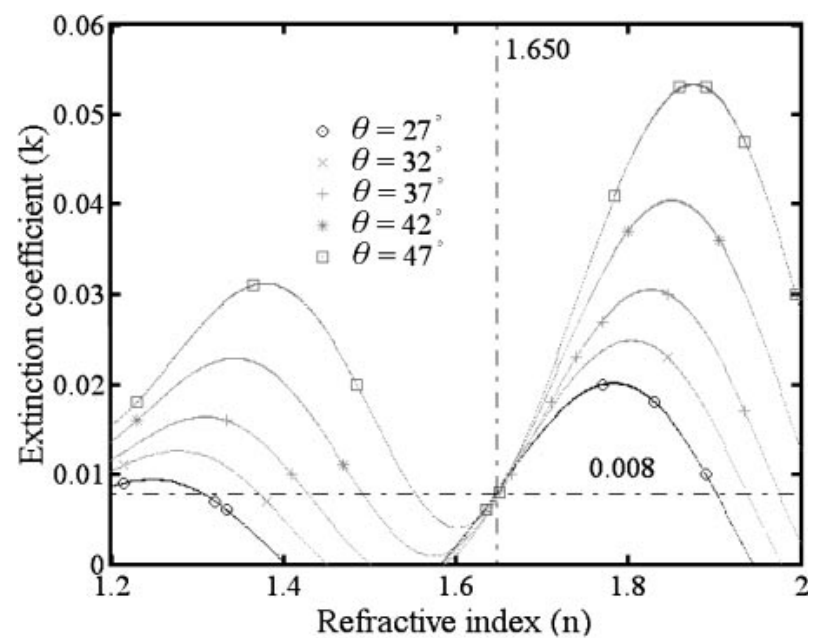

Fig. 6. Optical constants of silicon oxide films obtained from reflectance with different incidence angles.

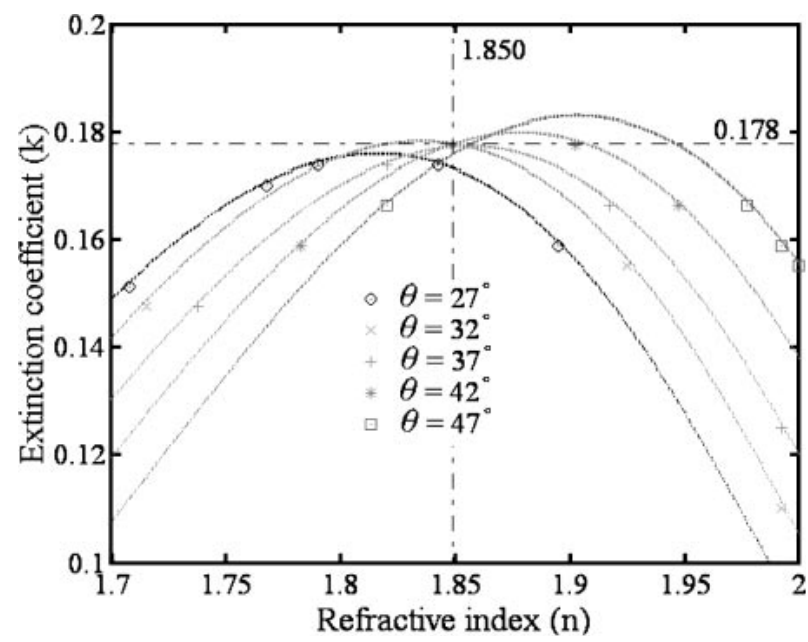

Fig. 7. Optical constants of silicon nitride films obtained from reflectance with different incidence angles.

Similarly, the measured surface roughness and thickness of a silicon nitride film were $0.369 \mathrm{~nm}$ and $83 \mathrm{~nm}$, respectively. By modifying the reflectance, Fig. 7 shows that the optical constants determined were about (1.850, $0.178)$. It was also demonstrated that the optical constants of semi-absorption materials can be obtained by this method.

For the high-absorption materials, the measured roughness of a thick titanium nitride film was $0.185 \mathrm{~nm}$ with the thickness larger than $100 \mathrm{~nm}$. As shown in Fig. 8, the optical constants obtained were about $(1.533,0.970)$. The result is similar to that of the optical constants of titanium nitride films in ref. 9. The results demonstrate that the optical constants of high-absorption materials can also be obtained by this method without obtaining the accurate thickness of thin films.

\section{Conclusions}

We demonstrate a simple method for determining optical constants in vacuum ultraviolet regimes. The optical constants were obtained by measuring the reflectance of 


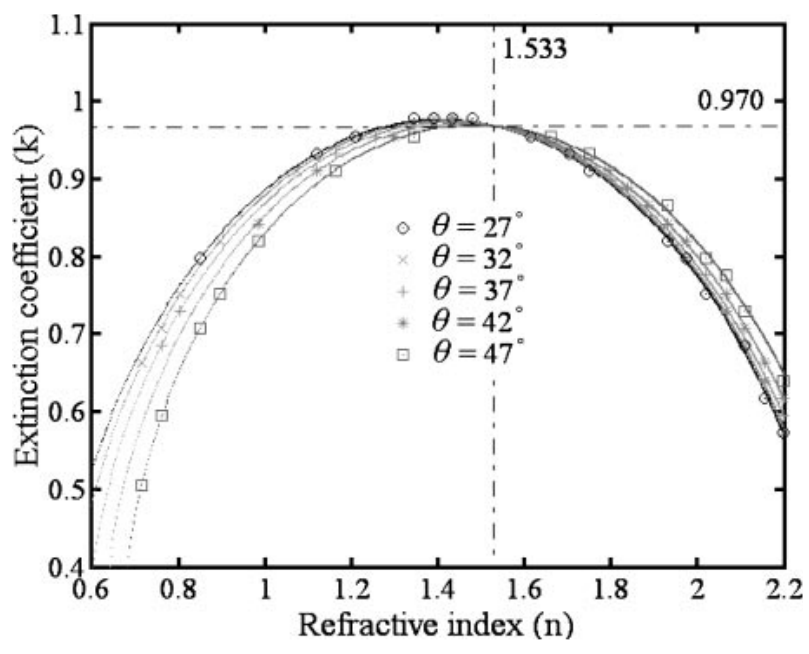

Fig. 8. Optical constants of titanium nitride films obtained from reflectance with different incidence angles.

thin films with different incidence angles using a vacuum ultraviolet spectrometer. In order to obtain accurate optical constants, the measurement of reflectance, thickness, and incidence angles should be affirmed carefully. In addition, it was determined that thickness measurement is not important for thick high-absorption films. We also determined that the measured reflectance should be modified by the surface roughness of thin films particularly in vacuum ultraviolet regimes. Optical constants of low-absorption, semi-absorption, and high-absorption materials can be obtained by this method experimentally without using complicated mathematical models. Therefore, this method can be used easily to determine optical constants of various materials for use in vacuum ultraviolet lithography.

\section{Acknowledgment}

The authors are very grateful to the National Science Council, Taiwan, R.O.C. for supporting the NSC92-2722-2317-200 project.

1) V. Liberman: J. Vac. Sci. \& Technol. B 20 (2002) 2567.

2) Semiconductor Industry Association, International Technology Roadmap for Semiconductor 2001 Updated.

3) T. C. Paulick: Appl. Opt. 25 (1986) 562.

4) N. V. Edwards: Annual APS March meet. 2002.

5) R. H. French: Proc. SPIE 4000 (2000) 1491.

6) W. R. Hunter: J. Opt. Soc. Am. 55 (1965) 1199.

7) J. M. Elson: App. Opt. 22 (1983) 3207.

8) H. A. Macleod: Thin Film Optical Filters (Macmillan, New York, 1986) p. 40.

9) B. W. Smith: J. Vac. Sci. \& Technol. B 20 (2002) 2578. 